CECS-PHY-11/07

\title{
Field theories with anisotropic scaling in 2D, solitons and the microscopic entropy of asymptotically Lifshitz black holes
}

\author{
Hernán A. González ${ }^{1}$, David Tempo ${ }^{2}$, Ricardo Troncoso ${ }^{2}$ \\ ${ }^{1}$ Departamento de Física, Pontificia Universidad Católica de Chile, \\ Casilla 306, Santiago 22, Chile. and \\ ${ }^{2}$ Centro de Estudios Científicos (CECs), Casilla 1469, Valdivia, Chile.
}

\begin{abstract}
Field theories with anisotropic scaling in $1+1$ dimensions are considered. It is shown that the isomorphism between Lifshitz algebras with dynamical exponents $z$ and $z^{-1}$ naturally leads to a duality between low and high temperature regimes. Assuming the existence of gap in the spectrum, this duality allows to obtain a precise formula for the asymptotic growth of the number of states with a fixed energy which depends on $z$ and the energy of the ground state, and reduces to the Cardy formula for $z=1$.

The holographic realization of the duality can be naturally inferred from the fact that Euclidean Lifshitz spaces in three dimensions with dynamical exponents and characteristic lengths given by $z, l$, and $z^{-1}, z^{-1} l$, respectively, are diffeomorphic. The semiclassical entropy of black holes with Lifshitz asymptotics can then be recovered from the generalization of Cardy formula, where the ground state corresponds to a soliton. An explicit example is provided by the existence of a purely gravitational soliton solution for BHT massive gravity, which precisely has the required energy that reproduces the entropy of the analytic asymptotically Lifshitz black hole with $z=3$.

Remarkably, neither the asymptotic symmetries nor central charges were explicitly used in order to obtain these results.
\end{abstract}




\section{INTRODUCTION}

Field theories with anisotropic scaling are of interest in a variety of subjects, specially in the context of quantum criticality in condensed matter physics (see e.g. [1, 2]). These theories have recently attracted the attention of the high energy physics community with the aim of finding a holographic description of the strong coupling regime, along the lines of the AdS/CFT correspondence [3-5]. In this sense, the so-called Lifshitz spacetimes, first introduced by Kachru, Liu and Mulligan in [6], were proposed as gravitational duals for which the anisotropic scaling symmetry is manifestly realized though its isometries ${ }^{1}$. As in the case of asymptotically AdS spacetimes [8], finite temperature effects are holographically introduced through a black hole in the bulk that asymptotically approaches to the Lifshitz spacetime. Since then, several asymptotically Lifshitz black hole solutions have been found, including analytic [9-19] and numerical results [20 26].

In two spacetime dimensions, field theories with anisotropic scaling have also been considered along different contexts in e.g., Refs. [27-29], and very recently in [30].

One of the main results reported here is that the semiclassical entropy of black holes with Lifshitz asymptotics can be obtained from the asymptotic growth of the number of states of a field theory with Lifshitz scaling in two dimensions, where the ground state in the bulk corresponds to a soliton.

The plan of the paper is as follows. In the next Section, field theories with Lifshitz scaling in $1+1$ dimensions are considered, and it is shown that a duality between high and low temperature regimes naturally arises as a consequence of the fact that Lifshitz algebras with dynamical exponents $z$ and $z^{-1}$ are isomorphic. In Section IIA, a precise formula for the asymptotic growth of the number of states with a fixed energy which depends on $z$ and the energy of the ground state is found, which reduces to the Cardy formula for $z=1$. The holographic realization of these results is carried out in Sec. III, where it is shown that the semiclassical entropy of black holes with Lifshitz asymptotics can then be recovered from the corresponding generalization of Cardy formula, provided the ground state is identified as a soliton. An explicit example is provided in Section IIIA, where a purely gravitational soliton solution for BHT massive gravity is found, which precisely has the required energy

\footnotetext{
${ }^{1}$ These spacetimes were previously discussed in the context of braneworlds in [7]. We thank Peter Koroteev for bringing this reference to our attention.
} 
that reproduces the entropy of the analytic asymptotically Lifshitz black hole with $z=3$ found in [12], while an exhaustive computation for this case is performed in Sec. IIIB, Finally, Section IV is devoted to the summary and the discussion.

\section{FIELD THEORIES WITH ANISOTROPIC SCALING IN 1+1 DIMENSIONS AT FINITE TEMPERATURE}

Let us consider a field theory in two spacetime dimensions with anisotropic scaling of the form,

$$
t \rightarrow \lambda^{z} t, x \rightarrow \lambda x
$$

This symmetry together with translations and shifts in time given by $x \rightarrow x+x_{0}$, and $t \rightarrow t+t_{0}$, are spanned by the following algebra

$$
[P, H]=0, \quad[D, P]=P, \quad[D, H]=z H,
$$

where $D$ is the generator of (1), and $P, H$ stand for the momentum generator and the Hamiltonian, respectively. This is known as a two-dimensional Lifshitz algebra with dynamical exponent given by $z$ (see e.g. [4, 31]).

A key observation that is worth to be stressed is the following: Lifshitz algebras of the form (2) with dynamical exponents $z$ and $z^{-1}$ are isomorphic. This can be easily verified by performing the following change of basis

$$
\bar{P}=H, \quad \bar{H}=P, \quad \bar{D}=z^{-1} D,
$$

so that the algebra (22) is mapped into

$$
[\bar{P}, \bar{H}]=0, \quad[\bar{D}, \bar{P}]=\bar{P}, \quad[\bar{D}, \bar{H}]=z^{-1} \bar{H} .
$$

Remarkably, this local isomorphism naturally induces the equivalence between the partition function at low and high temperatures for the same theory on a cylinder of radius $l$. This can be seen as follows. At finite temperature $T=\beta^{-1}$ the partition function can be defined on a torus where the Euclidean time $(\tau=i t)$ is periodic, such that $0 \leq \tau<\beta$, and $0 \leq \phi<2 \pi l$. Therefore, the change of basis (31) swaps the role of Euclidean time and the angle, so that their periods would be $0 \leq \bar{\phi}<\beta$, and $0 \leq \bar{\tau}<2 \pi l$. The period of the angle is then restored by virtue of scaling generated by $\bar{D}$ in (4), given by

$$
\bar{\tau} \rightarrow \lambda^{\frac{1}{z}} \bar{\tau}, \bar{\phi} \rightarrow \lambda \bar{\phi}
$$


with $\lambda=2 \pi l \beta^{-1}$. Hence the period of $\bar{\tau}$ become related with the period of $\tau$ according to

$$
\bar{\beta}=(2 \pi l)^{1+\frac{1}{z}} \beta^{-\frac{1}{z}}
$$

which allows to establish the relationship between the partition function at low and high temperatures as $Z[\bar{\beta}]=Z[\beta]$. In sum, the partition function can be assumed to be invariant under

$$
Z[\beta]=Z\left[(2 \pi l)^{1+\frac{1}{z}} \beta^{-\frac{1}{z}}\right],
$$

which for $z=1$ reduces to the well known $S$-modular invariance for chiral movers in conformal field theory [32].

\section{A. Asymptotic growth of the number of states}

Assuming the existence of gap in the spectrum, the duality of low and high temperatures regimes of the partition function (7) allows to obtain a precise formula for the asymptotic growth of the number of states with a fixed energy which depends on $z$ and the energy of the ground state, which is assumed to be negative and given by $-\Delta_{0}$.

The gap guarantees that at low temperatures the partition function becomes dominated by the contribution of the ground state, i.e., the partition function can be approximated as $Z[\beta] \approx e^{\beta \Delta_{0}}$. By virtue of Eq. (17) it follows that at high temperature the partition function is given by $Z[\beta] \approx e^{(2 \pi l)^{1+\frac{1}{z}} \beta^{-\frac{1}{z}} \Delta_{0}}$, and therefore, the asymptotic growth of the number of states at fixed energy $\Delta \gg \Delta_{0}$ can be obtained from

$$
\begin{aligned}
\rho(\Delta) & =\frac{1}{2 \pi i} \int d \beta Z[\beta] e^{\beta \Delta}, \\
& \approx \frac{1}{2 \pi i} \int d \beta e^{f(\beta, \Delta)},
\end{aligned}
$$

with $f(\beta, \Delta):=(2 \pi l)^{1+\frac{1}{z}} \beta^{-\frac{1}{z}} \Delta_{0}+\beta \Delta$. This expression can be evaluated in the saddle point approximation which is characterized by the extremum of $f(\beta, \Delta)$. This point corresponds to $\beta_{*}=2 \pi l\left(\frac{\Delta_{0}}{z \Delta}\right)^{\frac{z}{z+1}}$, and is such that $\left.\partial_{\beta} f\right|_{\beta_{*}}=0$. The entropy is then given by $S=$ 
$\log \rho(\Delta) \approx f\left(\beta_{*}, \Delta\right)$ which reads ${ }^{2}$

$$
S=2 \pi l(z+1)\left[\left(\frac{\Delta_{0}}{z}\right)^{z} \Delta\right]^{\frac{1}{z+1}} .
$$

Note that for $z=1$, the entropy reduces to $S=4 \pi l \sqrt{\Delta_{0} \Delta}$, so that Cardy formula is recovered $^{3}$. Moreover, the entropy in (99) fulfills the property $S\left(\Delta_{0}, \Delta, z\right)=S\left(\Delta, \Delta_{0}, z^{-1}\right)$, which is a consequence of the isomorphism of the Lifshitz algebras (21) and (44).

In the Canonical ensemble, the first law, $d \Delta=T d S$, allows to express the energy in terms of $\Delta_{0}$ and the temperature according to

$$
\Delta=\frac{1}{z}(2 \pi l)^{1+\frac{1}{z}} \Delta_{0} T^{1+\frac{1}{z}}
$$

which reduces to the Stefan-Boltzmann law for $z=1$. Analogously, the entropy can be written as

$$
S=(2 \pi l)^{1+\frac{1}{z}}\left(1+\frac{1}{z}\right) \Delta_{0} T^{\frac{1}{z}} .
$$

In what follows it is shown that the thermodynamics of three-dimensional black holes with Lifshitz asymptotics precisely fits the results found in this section, where the ground state turns out to be a soliton.

\section{ANISOTROPIC HOLOGRAPHY, SOLITONS AND THE MICROSCOPIC ENTROPY OF ASYMPTOTICALLY LIFSHITZ BLACK HOLES}

The holographic realization of the duality expressed by Eq. (7) can be naturally inferred from the fact that Euclidean Lifshitz spaces in three dimensions with dynamical exponents and characteristic lengths given by $z, l$, and $z^{-1}, z^{-1} l$, respectively, are diffeomorphic provided their Euclidean time periods are related exactly as in Eq. (6). This can be seen as

\footnotetext{
${ }^{2}$ We have implicitly assumed that the ground state is non degenerate; otherwise the asymptotic growth of the number of states is given by $\rho(\Delta)=\rho\left(\Delta_{0}\right) \exp \left[2 \pi l(z+1)\left[\left(\frac{\Delta_{0}}{z}\right)^{z} \Delta\right]^{\frac{1}{z+1}}\right]$, where $\rho\left(\Delta_{0}\right)$ stands for the ground state degeneracy.

${ }^{3}$ As explained in 33], in terms of the shifted Virasoro operator $\tilde{L}_{0}=L_{0}-\frac{c}{24}$, Cardy formula can be expressed only through its fixed and lowest eigenvalues, given by $\tilde{\Delta}$ and $-\tilde{\Delta}_{0}$, respectively. Thus, the asymptotic growth of the number of states can be obtained from the spectrum without making any explicit reference to the central charges. The corresponding energies are then given by $\Delta=\tilde{\Delta} l^{-1}$ and $-\Delta_{0}=-\tilde{\Delta}_{0} l^{-1}$, respectively.
} 
follows. The metric of Euclidean Lifshitz space in three dimensions is given by [6]

$$
d s^{2}=\frac{r^{2 z}}{l^{2 z}} d \tau^{2}+l^{2} \frac{d r^{2}}{r^{2}}+\frac{r^{2}}{l^{2}} d \phi^{2}
$$

where $0 \leq \tau<\beta$ and $0 \leq \phi<2 \pi l$. Performing a coordinate transformation defined by

$$
\bar{\phi}=\frac{2 \pi l}{\beta} \tau, \quad \bar{\tau}=\left(\frac{2 \pi l}{\beta}\right)^{\frac{1}{z}} \phi, \quad \bar{r}=\frac{\beta}{2 \pi z} \frac{r^{z}}{l^{z}},
$$

makes the line element (12) to acquire the form

$$
d s^{2}=\frac{z^{\frac{2}{z}}}{l^{\frac{2}{z}}} \bar{r}^{\frac{2}{z}} d \bar{\tau}^{2}+\frac{l^{2}}{z^{2}} \frac{d \bar{r}^{2}}{\bar{r}^{2}}+\frac{z^{2}}{l^{2}} \bar{r}^{2} d \bar{\phi}^{2},
$$

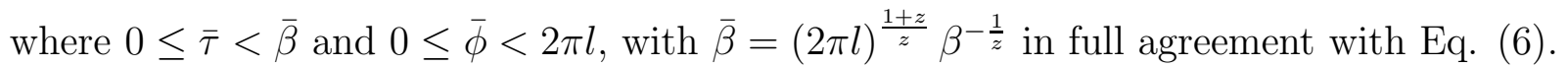

It is worth pointing out that, since this procedure is purely geometrical, the result remains valid regardless the theory under consideration. Therefore, analogously, an asymptotically Lifshitz Euclidean black hole with finite temperature $\beta$ in three dimensions becomes diffeomorphic to a soliton with temperature $\bar{\beta}$, with dynamical exponents and characteristic lengths given by $z, l$, and $z^{-1}, z^{-1} l$, respectively. The Lorentzian soliton is then regular everywhere and devoid of closed timelike curves provided $\bar{t}=i \bar{\tau}$ is unwrapped. As a consequence the soliton has a fixed mass, since the corresponding integration constant is reabsorbed by a simple rescaling. It becomes then natural to regard the soliton as the corresponding ground state.

Indeed, there are known examples for asymptotically AdS black holes and solitons in three dimensions where this mechanism successfully reproduces the microscopic entropy of the black hole by means of Cardy formula. This has been explicitly shown for a different class of black holes with scalar hair and scalar solitons in General Relativity [33], 34], as well as for hairy black holes and solitons in vacuum for BHT massive gravity [35-37]. In this sense, the soliton plays the role of AdS in General Relativity [32, 38, 39], since Euclidean AdS and the Euclidean BTZ black hole [40, 41] are diffeomorphic [42, 43].

Here we show how this mechanism can be extended in the case of asymptotically Lifshitz black holes and solitons in three dimensions.

Let us consider a soliton with mass given by $M_{\text {sol }}=-M_{0}$, with $M_{0}>0$, so that its Euclidean action reads

$$
I_{0}=\bar{\beta} M_{0}
$$


By virtue of the duality between low and high temperatures (6) the value of the Euclidean action for the corresponding black hole is then given by

$$
I=M_{0}(2 \pi l)^{1+\frac{1}{z}} \beta^{-\frac{1}{z}}
$$

Therefore, the black hole mass and its semiclassical entropy, given by $M=-\partial_{\beta} I, S=$ $\left(1-\beta \partial_{\beta}\right) I$, respectively, turn out to be

$$
M=\frac{1}{z}(2 \pi l)^{1+\frac{1}{z}} M_{0} T^{1+\frac{1}{z}}
$$

and

$$
S=(2 \pi l)^{1+\frac{1}{z}}\left(1+\frac{1}{z}\right) M_{0} T^{\frac{1}{z}} .
$$

Remarkably, the black hole mass (17) and entropy (18) precisely agree with the corresponding energy and entropy for the field theory with dynamical exponent $z$ in $1+1$ dimensions, given by (10) and (11), respectively, provided the black hole mass is given by the energy of the field theory, and the energy of the ground state is fixed by the soliton mass, i.e., for $\Delta_{0}=M_{0}$, and $\Delta=M$.

It is then reassuring to verify that once formula (17) is plugged into (18), one obtains

$$
S=2 \pi l(z+1)\left[\left(\frac{M_{0}}{z}\right)^{z} M\right]^{\frac{1}{z+1}}
$$

in full agreement with the asymptotic growth of the number of states in the corresponding field theory, given by (9))

For simplicity, we have considered here asymptotically Lifshitz spacetimes whose only nonvanishing global charge is the mass. In presence of additional global charges, one should work in the grand canonical ensemble.

An explicit example of these results is provided by the existence of a purely gravitational soliton solution for BHT massive gravity, which precisely has the energy that successfully reproduces the entropy of the analytic asymptotically Lifshitz black hole with $z=3$ found in Ref. [12]. This is discussed next. 


\section{A. An explicit example for BHT massive gravity}

The new massive gravity theory, recently proposed by Bergshoeff, Hohm and Townsend (BHT) is described by the following action [44]

$$
I_{\mathrm{BHT}}=-\frac{1}{16 \pi G} \int_{\mathcal{M}} d^{3} x \sqrt{-g}\left[R-2 \lambda-\frac{1}{m^{2}}\left(R_{\mu \nu} R^{\mu \nu}-\frac{3}{8} R^{2}\right)\right] .
$$

Hereafter we will focus on a special case, given by

$$
\lambda=13 m^{2}
$$

for which the theory admits Lifshitz spacetimes as vacuum solutions with dynamical exponents and characteristic lengths $z, l$ and $z^{-1}, z^{-1} l$, where $z=3$ and $l^{2}=-\frac{1}{2 m^{2}}$. As pointed out in [12], in this case the theory also admits a remarkably simple analytic asymptotically Lifshitz black hole solution in vacuum with $z=3$, whose metric reads

$$
d s^{2}=-\frac{r^{6}}{l^{6}}\left(1-\frac{r_{+}^{2}}{r^{2}}\right) d t^{2}+\frac{l^{2}}{r^{2}}\left(1-\frac{r_{+}^{2}}{r^{2}}\right)^{-1} d r^{2}+\frac{r^{2}}{l^{2}} d \phi^{2} .
$$

The coordinates range as $-\infty<t<+\infty, 0<r<\infty, 0 \leq \phi<2 \pi l$, and the event horizon is located at $r=r_{+}$, surrounding the singularity at the origin. Its Hawking temperature is then given by

$$
T=\beta^{-1}=\frac{1}{2 \pi l}\left(\frac{r_{+}}{l}\right)^{3}
$$

\section{Gravitational Soliton}

The theory (20) at the special case (21) also turns out to admit a gravitational soliton in vacuum. The solution is given by

$$
d s^{2}=-\cosh ^{2} \rho d \bar{t}^{2}+l^{2} d \rho^{2}+\cosh ^{4} \rho \sinh ^{2} \rho d \bar{\phi}^{2}
$$

with $-\infty<\bar{t}<+\infty, 0 \leq \rho<\infty$, and $0 \leq \bar{\phi}<2 \pi l$, and the scalar curvature reads

$$
R=-\frac{18}{l^{2}}-\frac{8}{l^{2}} \tanh ^{2} \rho
$$

This spacetime is regular everywhere, geodesically complete and shares the same causal structure with AdS spacetime. The soliton describes an asymptotically Lifshitz spacetime 
with dynamical exponent $z=\frac{1}{3}$ and characteristic length $\frac{l}{3}$, as it can be explicitly verified performing a redefinition of the radial coordinate according to

$$
\bar{r}=\frac{l}{3} \cosh ^{3} \rho,
$$

so that for $\bar{r} \rightarrow \infty$, the metric (24) approaches to that of a (Lorentzian) Lifshitz spacetime (12) with the corresponding dynamical exponent and characteristic length.

It is worth highlighting that, unlike the Lifshitz spacetime (12), the soliton (24) is devoid of divergent tidal forces near the origin.

It is then simple to verify that the Euclidean continuation of the soliton (24) with $\bar{\tau}=i \bar{t}$, is diffeomorphic to the Euclidean black hole (22) with $\tau=i t$, provided their temperatures are related as in Eq. (6). The corresponding coordinate transformation is given by (13), which in order to fit the gauge choice of the radial coordinate as in Eq. (24), has to be followed by (25).

In sum, the theory (20) at the special case (21) admits nontrivial asymptotically Lifshitz solutions in vacuum, given by the black hole (22) and the gravitational soliton (24). Note that since their corresponding dynamical exponents and characteristic lengths are given by $3, l$, and $\frac{1}{3}, \frac{l}{3}$, respectively, both metrics do not match at infinity, which a priori may appear as an obstacle to compare them in the same footing. Indeed, the obstruction remains for asymptotically Lifshitz spacetimes with generically different dynamical exponents and characteristic lengths. Nonetheless, and remarkably, when they are related as $z, l$, and $z^{-1}$, $z^{-1} l$, as in our case, the obstacle can be circumvented due to the fact that their corresponding Euclidean continuations are diffeomorphic provided the temperatures are related according to Eq. (6), and hence, any suitably regularized Euclidean action for the black hole is necessarily finite for the gravitational soliton and vice versa. This is explicitly confirmed in the next subsection.

\section{B. Regularized Euclidean action: Soliton as the ground state and microscopic}

\section{black hole entropy}

The BHT action (20) at the special point (21) can be regularized following the quasilocal approach of Brown and York [45], endowed with suitable counterterms along the lines of 
[46, 47]. For the case under consideration this task was carried out by Hohm and Tonni in [48], where it was shown that the regularized Brown-York stress energy tensor gives the mass for the asymptotically Lifshitz black hole (22) which agrees with the first law of thermodynamics. Here we show that the regularized Euclidean action also reproduces the expected results, as in Eqs. (15) and (16), for the gravitational soliton (24) and the black hole (22), respectively.

In second order formalism the bulk action (20) can be expressed as

$$
I_{\mathrm{BHT}}=-\frac{1}{16 \pi G} \int_{\mathcal{M}} d^{3} x \sqrt{-g}\left[R-2 \lambda-f^{\mu \nu} G_{\mu \nu}+\frac{1}{4} m^{2}\left(f_{\mu \nu} f^{\mu \nu}-f^{2}\right)\right],
$$

where $f^{\mu \nu}$ is an auxiliary field. The regularized action is given by [48]

$$
I_{\text {reg }}=I_{\mathrm{BHT}}+I_{\mathrm{GH}}+I_{\mathrm{ct}}
$$

where the boundary terms read

$$
\begin{aligned}
I_{\mathrm{GH}} & =-\frac{1}{16 \pi G} \int_{\partial \mathcal{M}} d^{2} x \sqrt{-\gamma}\left[-2 K-\hat{f^{i j}} K_{i j}+\hat{f} K\right], \\
I_{\mathrm{ct}} & =\frac{1}{32 \pi G l^{2}} \int_{\partial \mathcal{M}} d^{2} x \sqrt{-\gamma}\left[15+\frac{1}{2} \hat{f}-\frac{1}{16} \hat{f}^{2}\right] .
\end{aligned}
$$

Here, $\hat{f}^{i j}$ is defined in terms of $f^{i j}$ and the shift $N^{j}$ of a radial ADM decomposition of the bulk metric according to

$$
\hat{f^{i j}}=f^{i j}+2 f^{r(i} N^{j)}+f^{r r} N^{i} N^{j} .
$$

\section{Gravitational Soliton}

Evaluating the Euclidean continuation of the gravitational soliton (24) on each term of the regularized action (27), one obtains that the relevant terms are given by

$$
I_{\text {ВНт }}=\frac{\bar{\beta}}{2 G}\left(-\frac{e^{2 \rho}}{2}+1\right), I_{\mathrm{GH}}=\frac{\bar{\beta}}{2 G} e^{2 \rho}, I_{\mathrm{ct}}=\frac{\bar{\beta}}{4 G}\left(-e^{2 \rho}+1\right),
$$

so that the divergences exactly cancel out and the total Euclidean action (27) for the soliton becomes finite and given by

$$
I_{0}=\frac{3}{4 G} \bar{\beta}
$$

Therefore, as expected, the soliton has a fixed negative mass is given by

$$
M_{s o l}=-M_{0}=-\partial_{\bar{\beta}} I_{0}=-\frac{3}{4 G} .
$$


As explained in section III, since the soliton (24) is diffeomorphic to the black hole (22), the Euclidean action of the latter is obtained from (32), by virtue of the relationship between their Euclidean time periods as in Eq. (6). Nevertheless, an explicit evaluation of the regularized Euclidean action turns out to be a reassuring and healthy exercise which is performed next.

\section{Asymptotically Lifshitz black hole}

Each term of the regularized action (27), once evaluated in the Euclidean continuation of the black hole (22), gives

$$
I_{\mathrm{BHT}}=\frac{\beta r_{+}^{2}}{G l^{4}}\left(-r^{2}+r_{+}^{2}\right), I_{\mathrm{GH}}=\frac{\beta r_{+}^{2}}{G l^{4}}\left(2 r^{2}-r_{+}^{2}\right), I_{\mathrm{ct}}=\frac{\beta r_{+}^{2}}{G l^{4}}\left(-r^{2}+\frac{3 r_{+}^{2}}{4}\right),
$$

and hence, divergences again cancel out so that the Euclidean action (27) is finite and reads

$$
I=\frac{3 \beta}{4 G} \frac{r_{+}^{4}}{l^{4}}
$$

Once expressed in terms of the temperature (23), the Euclidean action (35) is given by

$$
I=\frac{3}{4 G}(2 \pi l)^{\frac{4}{3}} \beta^{\frac{-1}{3}}
$$

which reduces to $-\beta$ times the free energy in the semiclassical approximation, i.e., $I=-\beta F$, with $F=M-T S$. Therefore, the first law is recovered requiring the action to possess an extremum, so that the mass, $M=-\partial_{\beta} I$, is given by

$$
M=\frac{1}{4 G}(2 \pi l)^{\frac{4}{3}} \beta^{-\frac{4}{3}}=\frac{1}{4 G}\left(\frac{r_{+}}{l}\right)^{4} .
$$

This is in agreement with the result found through the evaluation of the Brown-York stressenergy tensor in [48]. The mass has also been computed by different methods in Refs. [49, 50]. The semiclassical black hole entropy is then given by $S=\left(1-\beta \partial_{\beta}\right) I$, which reduces to

$$
S=\frac{(2 \pi l)^{\frac{4}{3}}}{G} \beta^{-\frac{1}{3}}=\frac{2 \pi r_{+}}{G},
$$

and agrees with the result found in [13, 48] by means of Wald's formula [51].

Note that by virtue of the precise value of the soliton mass in Eq. (33), the Euclidean action, mass and black hole entropy obtained in the previous section, given by the generic 
formulae (16), (17) and (18), exactly reduce to the ones computed above, given by (35), (37) and (38), respectively.

The link with the microscopic counting of states of the field theory with anisotropic scaling is then seen as follows. Once formula (37) is plugged into (38) one obtains the black hole entropy as a function of its mass, given by

$$
S=8 \pi l\left[\left(\frac{1}{4 G}\right)^{3} M\right]^{\frac{1}{4}}
$$

which precisely agrees with (19), and hence with and the asymptotic growth of the number of states in Eq. (9), provided the black hole mass is identified with the energy of the dual field theory, and the energy of the ground state is determined by the soliton mass, i.e., for $\Delta_{0}=M_{0}=\frac{3}{4 G}$ and $\Delta=M$.

\section{SUMMARY AND DISCUSSION}

Field theories with anisotropic scaling of the form (11) in $1+1$ dimensions were analyzed. It was shown that the isomorphism between Lifshitz algebras (2) and (41) with dynamical exponents $z$ and $z^{-1}$, respectively, naturally induces the equivalence between the partition function at high and low temperatures for the same theory on a cylinder of radius $l$, according to Eq. (17). In the case of $z=1$ it is simple to verify that the Lifshitz algebra (2) is isomorphic to Poincaré in $1+1$, and it is then amusing to verify that the well-known $S$ modular invariance for chiral movers in conformal field theory can be recovered requiring much less symmetries. The existence of a gap in the spectrum guarantees that at low temperatures the partition function becomes dominated by the contribution of the ground state. Hence, the duality allows to obtain a precise formula for the asymptotic growth of the number of states with a fixed energy, given by (9), which depends on $z$ and the energy of the ground state, and reduces to Cardy formula for $z=1$. In the canonical ensemble, the energy and the entropy acquire the expected dependence on the temperature for a field theory with the anisotropic scaling (1) (see e.g. [9], [52]), and remarkably their precise form, which gives a measure of the number of degrees of freedom, becomes determined by the dynamical exponent $z$ and the ground state energy $-\Delta_{0}$ as in Eqs. (10) and (11), respectively.

The duality expressed by Eq. (7) admits an interesting holographic realization. This can be seen from the fact that Euclidean Lifshitz spaces in three dimensions (12) and (14) with 
dynamical exponents and characteristic lengths given by $z, l$, and $z^{-1}, z^{-1} l$, respectively, are related by the coordinate transformation (13), so that they are diffeomorphic provided their Euclidean time periods are related exactly as in Eq. (6).

Remarkably, this procedure is purely geometrical, and hence the result remains the same regardless the theory under consideration. As a consequence, the semiclassical entropy of black holes with Lifshitz asymptotics can then be recovered from the generalization of Cardy formula in (9), where the ground state corresponds to a soliton. This result stems from the fact that an asymptotically Lifshitz Euclidean black hole in three dimensions and a soliton, with dynamical exponents and characteristic lengths given by $z, l$, and $z^{-1}, z^{-1} l$, respectively, become diffeomorphic provided their Euclidean time periods are related as in Eq. (6). In particular, in the sense of Horava and Melby-Thompson [53], the anisotropic conformal boundary of the Euclidean black hole and the soliton is then the same torus.

The Euclidean action of the black hole (16) is then related to the one of the soliton (15) by virtue of the duality between high and low temperatures (66), and therefore, the black hole entropy is shown to be given by (19). Remarkably, this formula precisely agrees with the asymptotic growth of the number of states in the corresponding field theory with dynamical exponent $z$ in $1+1$ dimensions, given by (9), provided the black hole mass is given by the energy of the field theory, and the energy of the ground state is fixed by the soliton mass.

Remarkably, neither the asymptotic symmetries nor central charges were explicitly used in order to obtain these results, and hence they should naturally extend for higher dimensional black holes with an emergent three-dimensional Lifshitz geometry near the horizon.

Since the soliton mass is negative, the specific heat of the black hole

$$
C=\frac{\partial M}{\partial T}=\frac{1+z}{z^{2}}(2 \pi l)^{1+\frac{1}{z}} M_{0} T^{\frac{1}{z}}
$$

is manifestly positive, and therefore the black hole may reach local thermal equilibrium with a heat bath. Nonetheless, at the critical temperature $T=\frac{1}{2 \pi l}$, which corresponds to the self-dual point of the transformation (6), i.e., $\bar{\beta}=\beta$, according to Eqs. (15) and (16), the soliton and the asymptotically Lifshitz black hole possess the same free energy. This means that at high temperatures the partition function turns out to be dominated by the black hole, while for low temperatures it becomes dominated by thermal radiation on the soliton. Note that at fixed temperature, apart from the black hole and the gravitational soliton, the 
Lifshitz spacetime also appears as an additional possible decay channel. However, since its free energy vanishes, the Lifshitz spacetime cannot dominate the partition function and it is then always unstable against thermal decay.

It is worth pointing out here that at an arbitrarily small temperature, since the horizon radius of the black hole becomes arbitrarily small, an external observer that is nearby the horizon may feel arbitrarily large tidal forces, as it occurs for a Lifshitz spacetime around the origin [6, 54, 55]. However, as explained above, since in this case the soliton is the preferred configuration, this mechanism can be regarded as a sort of extension of cosmic censorship that resolves the potential singularities in the tidal forces. Indeed, unlike Lifshitz spacetime, the Lorentzian soliton is geodesically complete and regular everywhere.

An explicit example of these results was provided by showing the existence of the gravitational soliton (24) for BHT massive gravity, which possesses the precise mass, given by (33), that successfully reproduces the entropy of the analytic asymptotically Lifshitz black hole with $z=3$ found in Ref. [12]. In this case, the soliton is an asymptotically Lifshitz spacetime with $z=\frac{1}{3}$. Note that for General Relativity, making use of "reverse engineering", i.e., plugging asymptotically Lifshitz metrics into the Einstein field equations in order to find the corresponding stress energy tensor, it has been shown that the null energy condition is violated for $z<1[56,57]$. It is then worth to remark that since the the gravitational soliton (24) is a solution of the field equations in vacuum, the whole spacetime is devoid of any kind of stress-energy tensor, and hence no energy conditions can be violated. Thus, the result found for General relativity does not apply for BHT massive gravity.

As an ending remark, it would be interesting to explore whether the soliton mass captures a possible central charge of an affine extension of the Lifshitz algebra (2).

Acknowledgments. We thank Pedro Alvarez, G. Barnich, Francisco Correa, Cristián Martínez, and specially to Stephane Detournay and Alfredo Pérez for many useful and enlightening discussions. This work has been partially funded by the Fondecyt grants $\mathrm{N}^{\circ}$ 1085322, 1095098, 3110141, and by the Conicyt grant ACT-91: "Southern Theoretical Physics Laboratory" (STPLab). H.G. thanks Conicyt for financial support. The Centro de Estudios Científicos (CECs) is funded by the Chilean Government through the Centers 
of Excellence Base Financing Program of Conicyt.

[1] J. A. Hertz, Phys. Rev. B14, 1165-1184 (1976).

[2] S. Sachdev, "Quantum Phase Transitions" (Cambridge University Press), (1999).

[3] C. P. Herzog, J. Phys. A A42, 343001 (2009). [arXiv:0904.1975 [hep-th]].

[4] S. A. Hartnoll, Class. Quant. Grav. 26, 224002 (2009) [arXiv:0903.3246 [hep-th]].

[5] J. Maldacena, arXiv:1106.6073 [hep-th]].

[6] S. Kachru, X. Liu, M. Mulligan, Phys. Rev. D78, 106005 (2008). arXiv:0808.1725 [hep-th]].

[7] P. Koroteev and M. Libanov, JHEP 0802, 104 (2008) [arXiv:0712.1136 [hep-th]].

[8] E. Witten, Adv. Theor. Math. Phys. 2, 505 (1998) arXiv:hep-th/9803131.

[9] M. Taylor, arXiv:0812.0530 [hep-th].

[10] E. J. Brynjolfsson, U. H. Danielsson, L. Thorlacius and T. Zingg, J. Phys. A 43, 065401 (2010) [arXiv:0908.2611 [hep-th]].

[11] K. Balasubramanian and J. McGreevy, Phys. Rev. D 80, 104039 (2009) arXiv:0909.0263 [hep-th]].

[12] E. Ayon-Beato, A. Garbarz, G. Giribet and M. Hassaine, Phys. Rev. D 80, 104029 (2009) arXiv:0909.1347 [hep-th]].

[13] R. G. Cai, Y. Liu and Y. W. Sun, JHEP 0910, 080 (2009) arXiv:0909.2807 [hep-th]].

[14] D. W. Pang, JHEP 1001, 116 (2010) [arXiv:0911.2777 [hep-th]].

[15] E. Ayon-Beato, A. Garbarz, G. Giribet and M. Hassaine, JHEP 1004, 030 (2010) arXiv:1001.2361 [hep-th]].

[16] M. H. Dehghani, R. B. Mann and R. Pourhasan, arXiv:1102.0578 [hep-th].

[17] W. Chemissany and J. Hartong, arXiv:1105.0612 [hep-th].

[18] H. Maeda and G. Giribet, arXiv:1105.1331 [gr-qc].

[19] J. Tarrio, S. Vandoren, arXiv:1105.6335 [hep-th]].

[20] U. H. Danielsson and L. Thorlacius, JHEP 0903, 070 (2009) [arXiv:0812.5088 [hep-th]].

[21] R. B. Mann, JHEP 0906, 075 (2009) [arXiv:0905.1136 [hep-th]].

[22] G. Bertoldi, B. A. Burrington and A. Peet, Phys. Rev. D 80, 126003 (2009) arXiv:0905.3183 [hep-th]].

[23] G. Bertoldi, B. A. Burrington and A. W. Peet, Phys. Rev. D 80, 126004 (2009) 
arXiv:0907.4755 [hep-th]].

[24] M. H. Dehghani and R. B. Mann, JHEP 1007, 019 (2010) arXiv:1004.4397 [hep-th]].

[25] W. G. Brenna, M. H. Dehghani and R. B. Mann, arXiv:1101.3476 [hep-th].

[26] I. Amado and A. F. Faedo, JHEP 1107, 004 (2011) arXiv:1105.4862 [hep-th]].

[27] A. V. Chubukov, S. Sachdev, T. Senthil, Nucl. Phys. B426, 601-643 (1994).

[28] K. Yang, Phys. Rev. Lett. 93 (2004) 066401.

[29] J. L. Cardy, Nucl. Phys. B 389, 577 (1993) arXiv:hep-th/9210002.

[30] D. M. Hofman and A. Strominger, arXiv:1107.2917 [hep-th].

[31] A. Adams, A. Maloney, A. Sinha, S. E. Vazquez, JHEP 0903, 097 (2009). arXiv:0812.0166 [hep-th]].

[32] J. L. Cardy, Nucl. Phys. B270, 186-204 (1986).

[33] F. Correa, C. Martinez and R. Troncoso, JHEP 1101, 034 (2011) arXiv:1010.1259 [hep-th]].

[34] M. Henneaux, C. Martinez, R. Troncoso and J. Zanelli, Phys. Rev. D 65, 104007 (2002) arXiv:hep-th/0201170].

[35] A. Perez, D. Tempo and R. Troncoso, arXiv:1106.4849 [hep-th].

[36] G. Giribet, J. Oliva, D. Tempo and R. Troncoso, Phys. Rev. D 80, 124046 (2009) arXiv:0909.2564 [hep-th]].

[37] J. Oliva, D. Tempo and R. Troncoso, JHEP 0907, 011 (2009) arXiv:0905.1545 [hep-th]].

[38] J. D. Brown and M. Henneaux, Commun. Math. Phys. 104, 207 (1986).

[39] A. Strominger, JHEP 9802, 009 (1998) arXiv:hep-th/9712251].

[40] M. Banados, C. Teitelboim and J. Zanelli, Phys. Rev. Lett. 69, 1849 (1992) arXiv:hep-th/9204099.

[41] M. Banados, M. Henneaux, C. Teitelboim and J. Zanelli, Phys. Rev. D 48, 1506 (1993) arXiv:gr-qc/9302012.

[42] S. Carlip and C. Teitelboim, Phys. Rev. D 51, 622 (1995) arXiv:gr-qc/9405070.

[43] J. M. Maldacena and A. Strominger, JHEP 9812, 005 (1998) arXiv:hep-th/9804085.

[44] E. A. Bergshoeff, O. Hohm and P. K. Townsend, Phys. Rev. Lett. 102, 201301 (2009) arXiv:0901.1766 [hep-th]].

[45] J. D. Brown and J. W. . York, Phys. Rev. D 47, 1407 (1993) arXiv:gr-qc/9209012.

[46] M. Henningson and K. Skenderis, JHEP 9807, 023 (1998) arXiv:hep-th/9806087.

[47] V. Balasubramanian and P. Kraus, Commun. Math. Phys. 208, 413 (1999) 
arXiv:hep-th/9902121.

[48] O. Hohm and E. Tonni, JHEP 1004, 093 (2010) [arXiv:1001.3598 [hep-th]].

[49] D. O. Devecioglu and O. Sarioglu, Phys. Rev. D 83, 124041 (2011) arXiv:1103.1993 [hep-th]].

[50] D. O. Devecioglu and O. Sarioglu, Phys. Rev. D 83, 021503 (2011) [arXiv:1010.1711 [hep-th]].

[51] R. M. Wald, Phys. Rev. D 48, 3427 (1993) arXiv:gr-qc/9307038.

[52] E. D'Hoker, P. Kraus, JHEP 1005, 083 (2010). arXiv:1003.1302 [hep-th]].

[53] P. Horava and C. M. Melby-Thompson, Gen. Rel. Grav. 43, 1391 (2011) arXiv:0909.3841 [hep-th]].

[54] G. T. Horowitz, S. F. Ross, Phys. Rev. D56, 2180-2187 (1997). hep-th/9704058].

[55] K. Copsey and R. Mann, JHEP 1103, 039 (2011) arXiv:1011.3502 [hep-th]].

[56] S. S. Gubser, A. Nellore, Phys. Rev. D80, 105007 (2009). arXiv:0908.1972 [hep-th]].

[57] C. Hoyos, P. Koroteev, Phys. Rev. D82, 084002 (2010). [arXiv:1007.1428 [hep-th]]. 